\title{
ROC analysis of selected matrix metalloproteinases (MMPs) and tissue inhibitors of metalloproteinases (TIMPs) in psoriatic patients
}

\author{
Edyta K. Glazewska ${ }^{1}$, Marek Niczyporuk' ${ }^{1}$, Slawomir Lawicki², Maciej Szmitkowski², Monika Zajkowska², \\ Magdalena Donejko', Andrzej Przylipiak ${ }^{1}$
}

${ }^{1}$ Department of Esthetic Medicine, Medical University of Bialystok, Bialystok, Poland

${ }^{2}$ Department of Biochemical Diagnostics, Medical University of Bialystok, Bialystok, Poland

Adv Dermatol Allergol 2018; XXXV (2): 167-173

DOI: https://doi.org/10.5114/pdia.2017.66621

\begin{abstract}
Introduction: Psoriasis is a common, chronic inflammatory disease characterised by typical scaly skin lesions. The role of matrix metalloproteinases (MMPs) and their tissue inhibitors (TIMPs) in the pathogenesis and development of this condition have been repeatedly emphasised in available literature.

Aim: ROC analysis of selected MMPs (MMP-2, MMP-3, MMP-9, MMP-12, TIMP-2) and TIMPs (TIMP-2, TIMP-3) in psoriasis patients. The area under the ROC curve (AUC) indicates the clinical usefulness of a biomarker and its diagnostic power.

Material and methods: Plasma samples of 49 patients suffering from plaque psoriasis and 40 healthy volunteers were evaluated. Concentrations of MMPs and TIMPs were determined using the enzyme-linked immunosorbent assay (ELISA) while Psoriasis Area and Severity Index (PASI) was used to define disease advancement.

Results: In the total psoriasis patients group, the largest area under the ROC curve was obtained for TIMP-3. After the division of the total group based on disease severity, the highest AUC of all tested parameters was observed for patients with mild disease severity and subgroup la for TIMP-3, for subgroup Ib for MMP-12, and for individuals with moderate disease severity for MMP-2. The combined analysis of all tested parameters showed an increase in AUC values in the total group examined as well as in all groups of disease severity.

Conclusions: These results indicate the usefulness and high diagnostic power of TIMP-3 in early detection of psoriasis. Additionally, the combination of all tested parameters appeared to be a valuable biomarker panel for the analysed disease.
\end{abstract}

Key words: MMP-2, MMP-3, MMP-9, MMP-12, TIMP-2, TIMP-3.

\section{Introduction}

Psoriasis is a chronic, immune-mediated skin disorder affecting approximately $3 \%$ of the global population. The pathogenesis of this multifactorial condition is still not entirely understood. A number of clinical and experimental studies have indicated that the presentation of psoriatic scales is the consequence of immune disturbances which result from complex interactions between genetic and environmental factors [1-4].

Crucial factors influencing the most appropriate therapy selection and successful alleviation of psoriasis symptoms, which significantly impair patients' quality of life, are a definitive diagnosis, the correct differentiation of clinical variants of the disease in their early stages, the determination of its advancement and activity [5, 6]. Considering the fact that psoriatic biomarkers presented in the available literature are not entirely specific or sensitive, researchers are attempting to find parameters whose levels would correlate more closely with disease presence and severity. We assumed that these parameters might include matrix metalloproteinase (MMP) and tissue inhibitors of matrix metalloproteinase (TIMPS).

Matrix metalloproteinases (MMPs) are a family of metallo-dependent $\left(\mathrm{Zn}^{2+}, \mathrm{Ca}^{2+}\right)$ endopeptidases which play a crucial role in the cleavage and remodelling of the extracellular matrix (ECM) and basal membrane (BM)

Address for correspondence: Slawomir Lawicki MD, Department of Biochemical Diagnostics, Medical University of Bialystok, 15 A Waszyngtona St, 15-267 Bialystok, Poland, phone: +48 857468 587, e-mail: slawicki@umb.edu.pl Received: 9.01.2017, accepted: 17.02.2017. 
components [7]. This important ability condition the implication of the analysed proteins in a number of physiological and pathological processes [8]. As reported in the available literature, MMPs are involved in the pathogenesis of psoriasis through the alteration of intracellular contacts, degradation of ECM and BM components, promotion of new vessels formation, and stimulation of immune cells infiltration [9]. Adverse effects of MMPs overexpression are suppressed by the tissue inhibitors of metalloproteinases (TIMPS). To date, four TIMPs have been identified in mammals [10].

Importantly, significant disturbances in the activity and concentration of the described endopeptidases and their tissue inhibitors have been detected during the process of psoriasis relapse and advancement [9].

As MMPs and TIMPs are believed to be involved in the etiopathogenesis and spread of psoriasis, the aim of the present study was to investigate the diagnostic power of selected factors in the detection of plaque psoriasis and assessment of disease activity and severity.

\section{Material and methods}

Table 1 shows the tested groups. The study included 49 chronic plaque psoriasis patients (early type) whose condition was diagnosed and treated in the Outpatient Clinic of the Department of Dermatology, University Hospital, Bialystok, Poland, between 2013 and 2015. Key exclusion criteria were as follows: the presence of arthritis, autoimmunological or chronic skin and systemic diseases, history or presence of malignancy. None of the patients was treated with systemic therapies within 12 weeks prior to the study. Moreover, no topical treatment had been given to subjects enrolled during the past 4 weeks.

Table 1. Characteristics of psoriasis patients and control group

\begin{tabular}{lcc}
\hline Study group & $\begin{array}{c}\text { Number } \\
\text { of patients }\end{array}$ \\
\hline Psoriasis patients: & $\begin{array}{c}49 \text { (23 females, } \\
26 \text { males })\end{array}$ \\
\hline Type & Plaque & 49 \\
\hline Median age (range) & $39(18-76)$ \\
\hline Severity & Mild & 34 \\
\cline { 2 - 3 } & Subgroup la (PASI < 5) & 15 \\
\cline { 2 - 3 } & Subgroup lb (PASI 5-10) & 19 \\
\cline { 2 - 3 } & Moderate & 15 \\
\cline { 2 - 3 } & PASI $>10-<30$ & 21 males $)$ \\
\hline Healthy subjects: & $36(18-75)$ \\
\hline Median age (range)
\end{tabular}

Participants were assigned to specific groups of disease severity on the basis of the Psoriasis Area and Severity Index (PASI) formulated by Fredrikson and Pettersson [11]. The PASI score varies from 0 to 72 (where score $10 \leq$ suggests mild psoriasis, score $>10<30$ - moderate psoriasis and score $\geq 30$ - severe psoriasis). Furthermore, patients with mild disease severity were divided into two subgroups la (PASI < 5) and Ib (PASI 5-10).

The mean duration of the disease in the analysed group was 18.33 years.

Forty age-, sex- and ethnicity-matched healthy controls, free of chronic inflammatory skin and systemic diseases, with no family history of psoriasis, were recruited from the general population.

All patients gave their informed consent to participation in the study. The research was approved by the Biochemical Committee of the Medical University of Bialystok, No. R-I-002/74/2014.

\section{Biochemical analysis}

Venous blood samples were collected from each patient into a heparin sodium tube, centrifuged for $15 \mathrm{~min}$ at $1000 \times \mathrm{g}$ to obtain plasma samples and stored at $-85^{\circ} \mathrm{C}$ until assayed. The tested parameters were measured with the enzyme-linked immunosorbent assay (ELISA) (MMP-2, MMP-3, MMP-9 and TIMP-2 with Quantikine Human HGFs Immunoassay, R\&D Systems, USA) (MMP-12 and TIMP-3 with Quantikine Human Immunoassay, Wuhan EIAab Science Co., Wuhan, China), according to the manufacturer's protocols. Duplicate samples were assessed for each patient.

The intra-assay coefficient of variation (CV\%) of MMP-3 is reported to be $6.1 \%$ at a mean concentration of $3.21 \pm 0.197 \mathrm{ng} / \mathrm{ml}$; MMP-9 to be $1.9 \%$ at a mean concentration of $2.04 \pm 0.039 \mathrm{ng} / \mathrm{ml}$, TIMP-3 to be $6.1 \%$; MMP-2 to be $3.6 \%$ at a mean concentration of 22.8 $\pm 0.828 \mathrm{ng} / \mathrm{ml}$; TIMP-2 to be $3.0 \%$ at a mean concentration of $6.09 \pm 0.181 \mathrm{ng} / \mathrm{ml}$; MMP-12 is reported to be $3.6 \%$ at a mean concentration of $22.8 \pm 0.828 \mathrm{ng} / \mathrm{ml}$.

The inter-assay coefficient of variation (CV\%) of MMP-3 is reported to be $7.0 \%$ at a mean concentration of $3.08 \pm 0.217 \mathrm{ng} / \mathrm{ml}$; MMP-9 to be $7.8 \%$ at a mean concentration of $2.35 \pm 0.184 \mathrm{ng} / \mathrm{ml}$; TIMP-3 to be $8.4 \%$; MMP2 to be $7.0 \%$ at a mean concentration of $21.5 \pm 1.51 \mathrm{ng} /$ $\mathrm{ml}$; TIMP-2 to be $7.3 \%$ at a mean concentration of 6.38 $\pm 0.467 \mathrm{ng} / \mathrm{ml}$; MMP-12 to be $7.0 \%$ at a mean concentration of $21.5 \pm 1.51 \mathrm{ng} / \mathrm{ml}$.

The assay showed no significant cross-reactivity or interference with numerous human cytokines and other growth factors.

\section{Statistical analysis}

Statistical analysis was performed using the Statistica 12.0 PL Program. We defined the receiver-operating characteristic (ROC) curve for all the tested parameters. 
The construction of the ROC curves was performed using the GraphROC program for Windows and the areas under ROC curve (AUCS) were calculated to evaluate the diagnostic accuracy and to compare AUCs for all the tested parameters separately and in combination. Statistically significant differences were defined as comparisons resulting in $p<0.05$.

The cut-off values were calculated using the Youden Index (as a criterion for selecting the optimum cut-off point) and for each of the tested parameters they were as follows: MMP-2 $116.38 \mathrm{ng} / \mathrm{ml}, \mathrm{MMP}-319.24 \mathrm{ng} / \mathrm{ml}$, MMP-9 122.23 ng/ml, MMP-12 4.75 ng/ml, TIMP-2 92.53 ng/ml, TIMP-3 449.34 ng/ml.

\section{Results}

Table 2 shows diagnostic sensitivity (DSe) and diagnostic specificity (DSp) of the investigated MMPs and TIMPs. We demonstrated that DSe of the tested parameters in the total psoriasis patients group was the highest for MMP-2 (100\%) and significantly higher than that for MMP-12 (75.5\%), TIMP-3 (71.4\%), MMP-3 (44.9\%), MMP-9 (34.7\%) and TIMP-2 (34.7\%). Out of all the parameters, the highest DSe was observed for MMP-2 in the group of individuals with mildly and moderately advanced psoriasis and subgroup lb (100\%, 93.3\%, 100\%, respectively). However MMP-2 DSp value was distinctly lower than that detected among other metalloproteinases and their tissue inhibitors in all the subgroups. Moreover, MMP-12 showed the highest DSe in subgroup la (86.7\%). The diagnostic
DSp of the tested parameters was the highest for MMP-9 in the total psoriasis patients group and individuals with moderately active disease (92.5\% in both cases), for TIMP-3 - in patients with mild scales and those from subgroup la (100\% in both cases), while for TIMP-2 - in the subjects comprising subgroup lb (100\%).

The combined analysis of all the parameters in the total group of psoriasis patients resulted in a significant increase in DSp (100\%). In all combinations, DSe decreased marginally in comparison with single parameter analysis.

The relationship between the diagnostic DSe and DSp is illustrated by the ROC curve. The area under the ROC curve (AUC) indicates the clinical usefulness of a biomarker and its diagnostic power. It also quantifies the overall ability of a test to differentiate between individuals with the disease and those without it. All data regarding AUCs at different stages of psoriasis advancement are presented in Table 3.

We noticed that the TIMP-3 area under the ROC curve (0.738; $p<0.001)$ in the total group of psoriasis patients was larger than the areas of all other MMPs and TIMPS, and statistically significantly larger in comparison to AUC $=0.5$, borderline of the diagnostic usefulness of a test ( $p=0.027$ in all cases except TIMP-2). The combined analysis of all the tested parameters in the total group of $B C$ resulted in a significant rise in AUCs (0.912, $p<0.001$ ) (Figure 1).

In patients with mild scales the highest AUC of all the tested parameters was found for TIMP-3 (0.785). Fur-

Table 2. Diagnostic criteria of tested parameters and in combined analysis

\begin{tabular}{|c|c|c|c|c|c|c|}
\hline \multirow{2}{*}{$\begin{array}{l}\text { Tested } \\
\text { parameters }\end{array}$} & \multirow{2}{*}{$\begin{array}{l}\text { Diagnostic } \\
\text { criteria (\%) }\end{array}$} & \multicolumn{5}{|c|}{ Psoriasis patients } \\
\hline & & Total group & Mild & Mild - subgroup la & Mild - subgroup Ib & Moderate \\
\hline \multirow[t]{2}{*}{ MMP-2 } & DSe & $100 \%$ & $100 \%$ & $80.0 \%$ & $100 \%$ & $93.3 \%$ \\
\hline & DSp & $37.5 \%$ & $37.5 \%$ & $57.5 \%$ & $45.0 \%$ & $72.5 \%$ \\
\hline \multirow[t]{2}{*}{ MMP-3 } & DSe & $44.9 \%$ & $38.2 \%$ & $33.3 \%$ & $42.1 \%$ & $60.0 \%$ \\
\hline & DSp & $85.0 \%$ & $85.0 \%$ & $85.0 \%$ & $85.0 \%$ & $85.0 \%$ \\
\hline \multirow[t]{2}{*}{ MMP-9 } & DSe & $34.7 \%$ & $52.9 \%$ & $46.7 \%$ & $47.4 \%$ & $46.7 \%$ \\
\hline & DSp & $92.5 \%$ & $72.5 \%$ & $92.5 \%$ & $72.5 \%$ & $92.5 \%$ \\
\hline \multirow[t]{2}{*}{ MMP-12 } & DSe & $75.5 \%$ & $73.5 \%$ & $86.7 \%$ & $68.4 \%$ & $80.0 \%$ \\
\hline & DSp & $65.0 \%$ & $65.0 \%$ & $60.0 \%$ & $65.0 \%$ & $70.0 \%$ \\
\hline \multirow[t]{2}{*}{ TIMP-2 } & DSe & $34.7 \%$ & $55.9 \%$ & $73.3 \%$ & $26.3 \%$ & $60.0 \%$ \\
\hline & DSp & $90.0 \%$ & $62.5 \%$ & $62.5 \%$ & $100.0 \%$ & $90.0 \%$ \\
\hline \multirow[t]{2}{*}{ TIMP-3 } & DSe & $71.4 \%$ & $55.9 \%$ & $80.0 \%$ & $52.6 \%$ & $73.3 \%$ \\
\hline & DSp & $70.0 \%$ & $100.0 \%$ & $100.0 \%$ & $85.0 \%$ & $57.5 \%$ \\
\hline \multirow{2}{*}{$\begin{array}{l}\text { MMP-2 } \\
+ \text { MMP-3 + } \\
\text { MMP-9 + MMP- } \\
12+\text { TIMP-2 + } \\
\text { TIMP-3 }\end{array}$} & DSe & $77.6 \%$ & $73.5 \%$ & $93.3 \%$ & $73.7 \%$ & $86.7 \%$ \\
\hline & DSp & $100.0 \%$ & $100.0 \%$ & $100.0 \%$ & $87.5 \%$ & $100.0 \%$ \\
\hline
\end{tabular}


Table 3. Diagnostic criteria of the ROC curve for tested parameters and in combined analysis

\begin{tabular}{|c|c|c|c|c|}
\hline Tested parameters & AUC & SE & $95 \% \mathrm{Cl}(\mathrm{AUC})$ & $p(\mathrm{AUC}=0.5)$ \\
\hline \multicolumn{5}{|c|}{ ROC criteria in psoriasis patients (total group): } \\
\hline MMP-2 & 0.656 & 0.0598 & $(0.539-0.773)$ & 0.009 \\
\hline MMP-3 & 0.631 & 0.0593 & $(0.515-0.747)$ & 0.027 \\
\hline MMP-9 & 0.641 & 0.0586 & $(0.526-0.756)$ & 0.016 \\
\hline MMP-12 & 0.688 & 0.0593 & $(0.572-0.804)$ & 0.002 \\
\hline TIMP-2 & 0.574 & 0.0612 & $(0.453-0.694)$ & 0.230 \\
\hline TIMP-3 & 0.738 & 0.0531 & $(0.634-0.842)$ & $<0.001$ \\
\hline $\begin{array}{l}\text { MMP-2 + MMP-3 } \\
+ \text { MMP-9 + MMP-12 + } \\
\text { TIMP-2 + TIMP-3 }\end{array}$ & 0.912 & 0.0322 & $(0.849-0.975)$ & $<0.001$ \\
\hline \multicolumn{5}{|c|}{ ROC criteria in psoriasis patients (mild cases): } \\
\hline MMP-2 & 0.562 & 0.0705 & $(0.424-0.700)$ & 0.381 \\
\hline MMP-3 & 0.583 & 0.0680 & $(0.449-0.716)$ & 0.224 \\
\hline MMP-9 & 0.619 & 0.0670 & $(0.488-0.750)$ & 0.075 \\
\hline MMP-12 & 0.672 & 0.0637 & $(0.547-0.797)$ & 0.007 \\
\hline TIMP-2 & 0.515 & 0.0711 & $(0.376-0.655)$ & 0.828 \\
\hline TIMP-3 & 0.785 & 0.0608 & $(0.665-0.904)$ & $<0.001$ \\
\hline $\begin{array}{l}\text { MMP-2 + MMP-3 + } \\
\text { MMP-9 + MMP-12 + } \\
\text { TIMP-2 + TIMP-3 }\end{array}$ & 0.892 & 0.0425 & $(0.809-0.976)$ & $<0.001$ \\
\hline \multicolumn{5}{|c|}{ ROC criteria in psoriasis patients (mild - subgroup la): } \\
\hline MMP-2 & 0.503 & 0.0852 & $(0.336-0.670)$ & 0.969 \\
\hline MMP-3 & 0.548 & 0.0900 & $(0.372-0.725)$ & 0.591 \\
\hline MMP-9 & 0.690 & 0.0868 & $(0.520-0.860)$ & 0.029 \\
\hline MMP-12 & 0.685 & 0.0759 & $(0.536-0.834)$ & 0.015 \\
\hline TIMP-2 & 0.612 & 0.0954 & $(0.425-0.799)$ & 0.242 \\
\hline TIMP-3 & 0.967 & 0.0215 & $(0.924-1.009)$ & $<0.001$ \\
\hline $\begin{array}{l}\text { MMP-2 + MMP-3 + } \\
\text { MMP-9 + MMP-12 + } \\
\text { TIMP-2 + TIMP-3 }\end{array}$ & 0.993 & 0.0074 & $(0.979-1.008)$ & $<0.001$ \\
\hline \multicolumn{5}{|c|}{ ROC criteria in psoriasis patients (mild - subgroup Ib): } \\
\hline MMP-2 & 0.613 & 0.0760 & $(0.464-0.762)$ & 0.136 \\
\hline MMP-3 & 0.610 & 0.0841 & $(0.445-0.775)$ & 0.191 \\
\hline MMP-9 & 0.563 & 0.0853 & $(0.396-0.730)$ & 0.459 \\
\hline MMP-12 & 0.662 & 0.0733 & $(0.518-0.805)$ & 0.027 \\
\hline TIMP-2 & 0.561 & 0.0874 & $(0.389-0.732)$ & 0.489 \\
\hline TIMP-3 & 0.641 & 0.0943 & $(0.456-0.826)$ & 0.135 \\
\hline $\begin{array}{l}\text { MMP-2 + MMP-3 + } \\
\text { MMP-9 + MMP-12 + } \\
\text { TIMP-2 + TIMP-3 }\end{array}$ & 0.813 & 0.0709 & $(0.674-0.951)$ & $<0.001$ \\
\hline \multicolumn{5}{|c|}{ ROC criteria in psoriasis patients (moderate cases): } \\
\hline MMP-2 & 0.870 & 0.0497 & $(0.773-0.967)$ & $<0.001$ \\
\hline MMP-3 & 0.740 & 0.0827 & $(0.578-0.902)$ & 0.004 \\
\hline MMP-9 & 0.690 & 0.0879 & $(0.518-0.862)$ & 0.031 \\
\hline MMP-12 & 0.725 & 0.0728 & $(0.582-0.868)$ & 0.002 \\
\hline TIMP-2 & 0.775 & 0.0752 & $(0.628-0.922)$ & $<0.001$ \\
\hline TIMP-3 & 0.632 & 0.0808 & $(0.473-0.790)$ & 0.103 \\
\hline $\begin{array}{l}\text { MMP-2 + MMP-3 + } \\
\text { MMP-9 + MMP-12 + } \\
\text { TIMP-2 + TIMP-3 }\end{array}$ & 0.957 & 0.0375 & $(0.883-1.030)$ & $<0.001$ \\
\hline
\end{tabular}




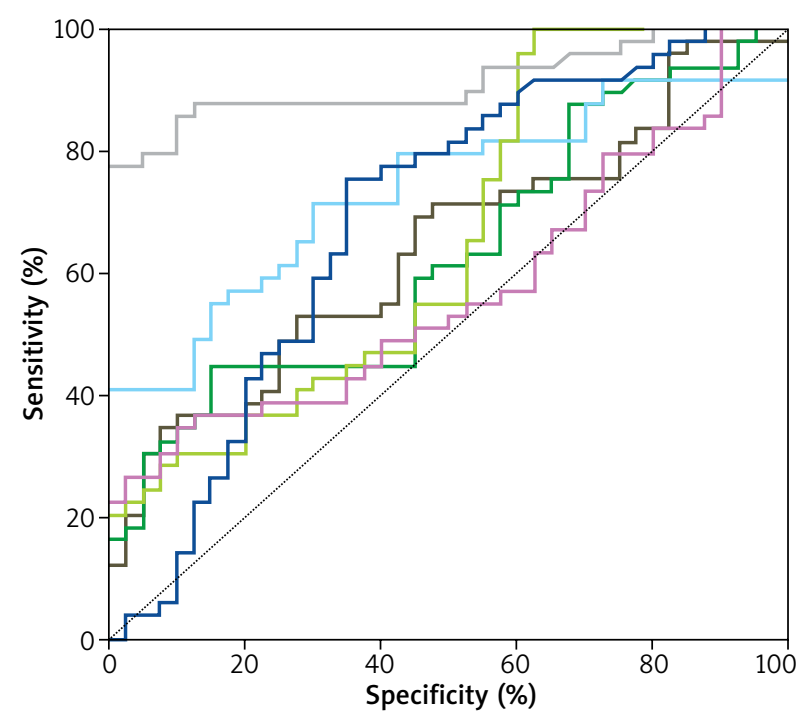

$$
\begin{array}{lll}
- \text { MMP-9 } & - \text { MMP-2 } & \text { - MMP-9 + MMP-3 + } \\
- \text { MMP-3 } & - \text { TIMP-2 } & \text { TIMP-3 + MMP- }+ \\
- \text { TIMP-3 } & - \text { MMP-12 } & \text { TIMP-2 + MMP-12 }
\end{array}
$$

Figure 1. Diagnostic criteria of the ROC curve for tested parameters and in combined analysis in the total psoriasis patients group

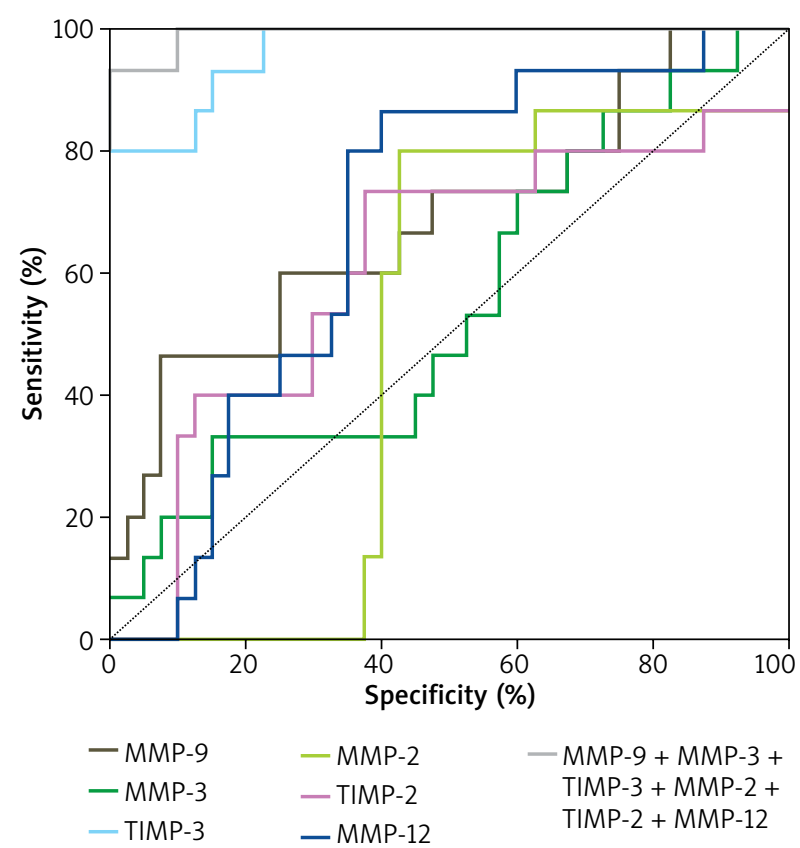

Figure 3. Diagnostic criteria of the ROC curve for tested parameters and in combined analysis in patients from subgroup la

thermore, only TIMP-3 and MMP-12 were statistically significantly larger in comparison to $\mathrm{AUC}=0.5(p=0.007$; $p<0.001)$ in those cases. The combined analysis of all the tested parameters showed a marginal increase in AUC (0.892; $p<0.001)$ (Figure 2).

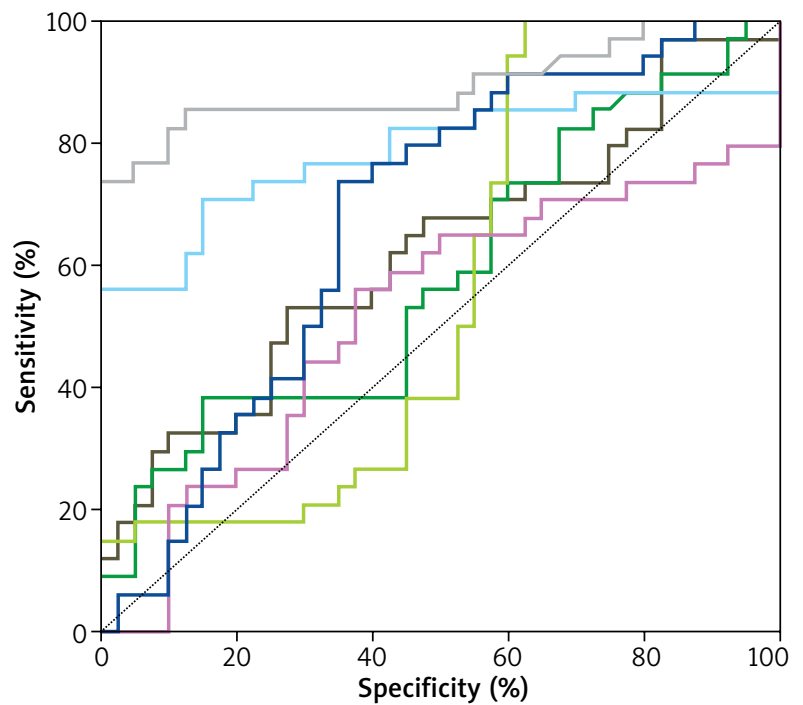

$\begin{array}{llr}- \text { MMP-9 } & - \text { MMP-2 } & \text { MMP-9 + MMP-3 + } \\ - \text { MMP-3 } & - \text { TIMP-2 } & \text { TIMP-3 + MMP-2 + } \\ - \text { TIMP-3 } & - \text { MMP-12 } & \text { TIMP-2 + MMP-12 }\end{array}$

Figure 2. Diagnostic criteria of the ROC curve for tested parameters and in combined analysis in subjects with a mild form of the disease

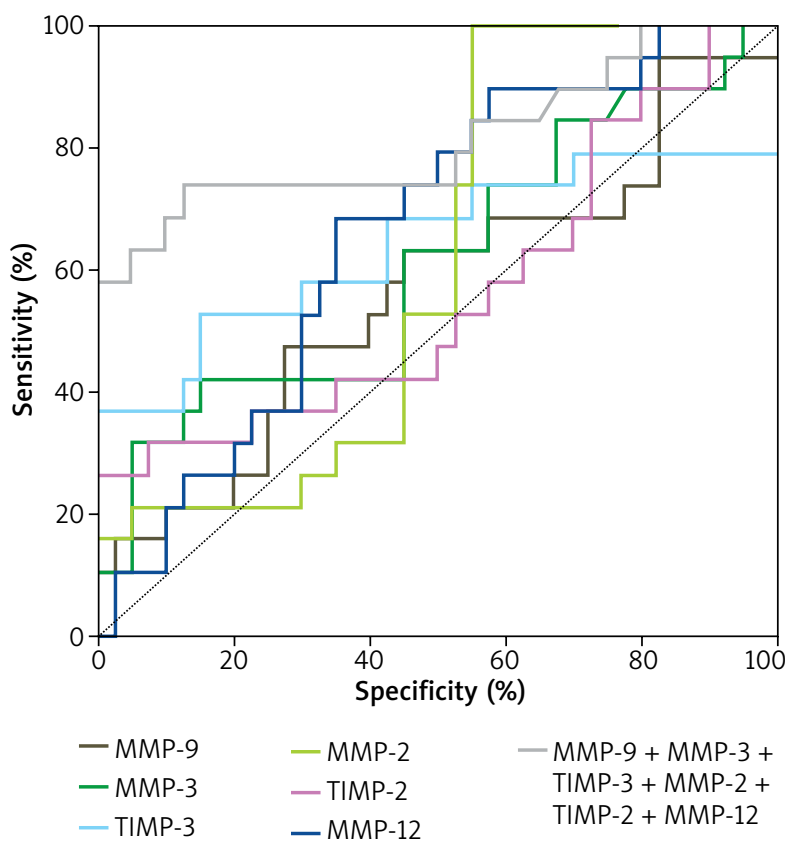

Figure 4. Diagnostic criteria of the ROC curve for tested parameters and in combined analysis in patients from subgroup Ib

In subgroup la of psoriasis patients, the highest AUC of all tested parameters was also observed for TIMP-3 (0.967, $p<0.001)$. The combined analysis of all the tested parameters showed a slight decrease in AUC (0.933; $p<0.001$ ) (Figure 3). 


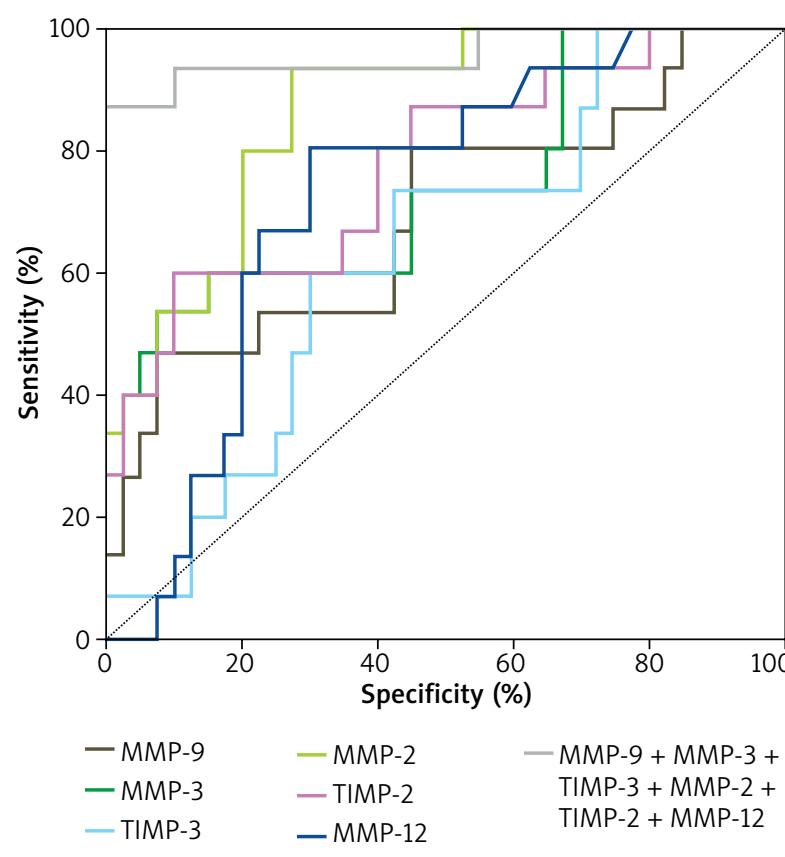

Figure 5. Diagnostic criteria of the ROC curve for tested parameters and in combined analysis in subjects with a moderate form of the disease

In subgroup Ib of psoriasis patients, the highest AUC of all the tested parameters was observed in MMP-12 (0.662, $p=0.027)$ and it was the only parameter which was statistically significantly larger in comparison to AUC $=0.5$. The combined analysis of all the tested parameters showed an increase in AUC values (0.813, $p<0.001$ ) (Figure 4).

In patients with moderately advanced disease, the highest AUC of all the tested parameters was found in MMP-2 (0.870, $p<0.001)$. The combined analysis of all the tested parameters showed an increase in AUC values (0.957, $p<0.001)$ (Figure 5).

\section{Disscussion}

Developing new plasma and serum biomarkers is critical for the provision of quantitative evidence of psoriasis presence in doubtful cases, determination of disease severity and management of patient treatment. A biomarker is an objective indicator of disease presence and advancement, or the effects of treatment, which can be measured accurately and consistently [12].

Numerous studies have demonstrated a variety of potential psoriasis biomarkers, i.e. cytokines, growth factors, hsCRP, ceruloplasmin, parameters of lipid profile, taurine, myo-inositol, choline, coagulation and fibrinosis parameters [5, 6, 13-15]. As reported in the available literature, genomic biomarkers of the condition have also been found [6].
Having analysed the results of studies performed to date, our research team decided to investigate MMPs and TIMPs as potential biomarkers of psoriasis. The endopeptidases are involved in the pathogenesis and spread of psoriasis thanks to their multifunctionality - from the degradation of ECM and BM components and altering intracellular contacts to the stimulation of inflammatory infiltration or angiogenesis [9]. TIMPs inhibit the activity of MMPs [16]. Furthermore, it has been demonstrated that in physiological conditions, the MMP: TIMP ratio is highly regulated. An imbalance in their proportion may lead to the pathological production and cleaving of ECM and BM components [17].

Our research performed to date has revealed significantly elevated plasma levels of MMP-2, MMP-3, MMP-9, TIMP-3, significantly decreased levels of MMP-12 and no significant changes in TIMP-2 concentration in the total psoriasis patients group when compared to the healthy individuals [18, 19]. Additionally, Flisiak et al. indicated significantly elevated concentrations of MMP-1 and TIMP-1 in the sera of psoriasis patients when compared to the control group [20]. A correlation between the concentration of the aforementioned MMPs and TIMPs and psoriasis advancement has also been revealed [18-20]. These findings indicate that the analysed factors appear to be valuable psoriasis biomarkers.

Due to a lack of studies documenting the diagnostic power of psoriasis biomarkers already presented in the literature, we decided to examine the diagnostic value of MMP-2, -3, -9, -12 and TIMP-2, -3 based on the ROC curve.

Diagnostic sensitivity (DSe) measures the proportion of positives that are correctly identified. In this study, DSe for MMP-2 was the highest in the total group of psoriasis patients as well as in mild and moderately severe cases. Simultaneously, DSp of this factor was the lowest among all the selected parameters, which means that with the use of plasma MMP-2 levels we are able to detect disease, but we are not certain if it is psoriasis.

The AUC represents the overall accuracy of a test, with the value approaching 1.0 indicating perfect DSe and DSp. According to the current study, the ROC area of TIMP-3 was the largest of all the tested parameters not only in the total group but also in mild cases and patients with fewer scaly lesions (PASI 0-5). The high AUC value for TIMP-3 in mild cases is a significant finding as it indicates the high usefulness of this factor in the early psoriasis diagnosis. Furthermore, we found a high value AUC area of MMP-2 in individuals with moderately severe disease. Moreover, the combined analysis of the tested parameters resulted in a significant increase in DSp and $A \cup C$ values in the total psoriasis patients group as well as in all subgroups of disease advancement, which proves that the combined analysis of selected MMPs and TIMP is a valuable biomarker panel of the described condition.

As previously highlighted, the available literature contains few papers describing the diagnostic power of pso- 
riasis markers. Hirao et al. demonstrated that hair root levels of miR-19a can effectively distinguish psoriasis patients from healthy individuals (AUC $=0.75)$ [21]. Furthermore, Chandran et al. indicated that increased levels of hsCRP, OPG, MMP-3 and the CPII : C2C ratio were independently associated with psoriatic arthritis and can differentiate patients with psoriasis alone from those with PsA with high power $(\mathrm{AUC}=0.94)$ [22].

Interestingly, the high diagnostic power of metalloproteinases and their tissue inhibitors in the detection of other disease, particularly cancer, have been repeatedly confirmed [23-25].

\section{Conclusions}

TIMP-3 was found to be a promising biomarker for the early detection of psoriasis. Furthermore, the combined analysis of all the tested MMPs and TIMPs proved to be a valuable diagnostic panel of this condition, respective of disease advancement.

\section{Acknowledgments}

This work was supported by a grant from the Medical University of Bialystok (N/ST/MN/16/001/2230).

\section{Conflict of interest}

The authors declare no conflict of interest.

\section{References}

1. Boehncke WH. Etiology and pathogenesis of psoriasis. Rheum Dis Clin North Am 2015; 41: 665-75.

2. Parisi R, Symmons DPM, Griffiths CEM, et al. Global epidemiology of psoriasis: a systematic review of incidence and prevalence. J Invest Dermatol 2013; 133: 377-85.

3. Cohen BE, Martires KJ, Ho RS. Psoriasis and the risk of depression in the US population: National Health and Nutrition Examination Survey 2009-2012. JAMA Dermatol 2016; 152: 73-9.

4. Huerta C, Rivero E, Rodríguez LA. Incidence and risk factors for psoriasis in the general population. Arch Dermatol 2007; 143: 1559-65.

5. Kang H, Li X, Zhou Q, et al. Exploration of candidate biomarkers for human psoriasis based on GC-MS serum metabolomics. Br J Dermatol 2017; 176: 713-22.

6. Jiang S, Hinchliffe TE, Wu T. Biomarkers of an autoimmune skin disease - psoriasis. Genomics Proteomics Bioinformatics 2015; 13: 224-33.

7. Nagase H, Woessner JF. Matrix metalloproteinases. J Biol Chem 1999; 274: 21491-4.

8. Ravi Kanth VV, Nageshwar Reddy D. Role of matrix metalloproteinases in physiological processes and disease. Indian J Med Res 2014; 140: 585-7.

9. Mezentsev A, Nikolaev A, Bruskin S. Matrix metalloproteinases and their role in psoriasis. Gene 2014; 540: 1-10.

10. Arpino V, Brock M, Gill SE. The role of TIMPs in regulation of extracellular matrix proteolysis. Matrix Biology 2015; 44-46: 247-54.
11. Fredriksson T, Pettersson U. Severe psoriasis oral therapy with a new retinoid. Dermatologica 1978; 157: 238-44.

12. Yamanaka K, Mizutani H. A new biomarker for psoriasis. Br J Dermatol 2016; 174: 1191-2.

13. Keerthana, BL, Kumar, TA. Serum biomarkers for diagnosis and assessment of severity in psoriasis. Int J Biomed Adv Res 2016; 7: 17-21.

14. Flisiak I, Zaniewski P, Chodynicka B. Plasma TGF-beta1, TIMP-1, MMP-1 and IL-18 as a combined biomarker of psoriasis activity. Biomarkers 2008; 13: 549-56.

15. Fotiadou C, Lazaridou E, Sotiriou E, et al. IL-17A, IL-22, and IL-23 as markers of psoriasis activity: a cross-sectional, hospital-based study. J Cutan Med Surg 2015; 19: 555-60.

16. Moore CS, Crocker SJ. An alternate perspective on the roles of TIMPs and MMPs in pathology. Am J Pathol 2012; 180: $12-6$.

17. Sartakhti JS, Manshaei MH, Sadeghi M. MMP-TIMP interactions in cancer invasion: an evolutionary game-theoretical framework. J Theor Biol 2017; 412: 17-26.

18. Głażewska EK, Niczyporuk M, Przylipiak A, et al. Influence of narrowband ultraviolet-B phototherapy on plasma concentration of matrix metalloproteinase-12 in psoriatic patients. Adv Dermatol Alergol 2017; 34: 328-33.

19. Głażewska EK, Niczyporuk M, Ławicki S, et al. Therapy of psoriasis with narrowband ultraviolet-B light influences plasma concentration of MMP-2 and TIMP-2 in patients. Ther Clin Risk Manag 2016; 21: 1579-85.

20. Flisiak I, Porebski P, Chodynicka B. Effect of psoriasis activity on metalloproteinase-1 and tissue inhibitor of metalloproteinase-1 in plasma and lesional scales. Acta Derm Venereol 2006; 86: 17-21.

21. Hirao H, Jinnin M, Ichihara A, et al. Detection of hair root miR19 a as a novel diagnostic marker for psoriasis. Eur J Dermatol 2013; 23: 807-11.

22. Chandran Y, Cook RJ, Edwin J, et al. Soluble biomarkers differentiate patients with psoriatic arthritis from those with psoriasis without arthritis. Rheumatology 2010; 49: 1399405.

23. Ricci S, Bruzzese D, DI Carlo A. Evaluation of MMP-2, MMP-9, TIMP-1, TIMP-2, NGAL and MMP-9/NGAL complex in urine and sera from patients with bladder cancer. Oncol Lett 2015; 10: 2527-32.

24. Ławicki S, Głażewska EK, Sobolewska M, et al. Plasma levels and diagnostic utility of macrophage colony-stimulating factor, matrix metalloproteinase-9, and tissue inhibitor of metalloproteinases-1 as new biomarkers of breast cancer. Ann Lab Med 2016; 36: 223-9.

25. Ławicki S, Zajkowska M, Głażewska EK, et al. Plasma levels and diagnostic utility of VEGF, MMP-2 and TIMP-2 in the diagnostics of breast cancer patients. Biomarkers 2017; 22: 157-64. 